Supplement of Atmos. Chem. Phys., 14, 6925-6940, 2014

http://www.atmos-chem-phys.net/14/6925/2014/

doi:10.5194/acp-14-6925-2014-supplement

(C) Author(s) 2014. CC Attribution 3.0 License.

(c) (1)

Supplement of

\title{
Factors controlling pollutant plume length downwind of major roadways in nocturnal surface inversions
}

W. Choi et al.

Correspondence to: S. E. Paulson (paulson@atmos.ucla.edu) 


\section{S1. Characteristics of sampling areas (Downtown LA, Paramount, Carson, and Claremont)}

To investigate the areal impact of freeway plumes on nearby residential neighborhoods under stable pre-sunrise conditions, four different measurement sites were selected in the South Coast Air Basin (SoCAB) in California: Downtown Los Angeles (DTLA), Paramount, Carson and Claremont (Fig. S1a). The SoCAB occupies a coastal plain surrounded by mountains on three sides (the San Gabriel, San Bernardino, and San Jacinto mountains). The predominant meteorological conditions in the SoCAB are characterized by mild winds and shallow boundary layer heights capped by low-altitude (500 to $1200 \mathrm{~m}$ above ground level) temperature inversions due to a semi-permanent "Pacific High" pressure cell. Prevailing winds are dominated by diurnal cycles of weak off-shore breezes at night and stronger on-shore sea breezes during the day. Nighttime surface cooling combined with weak winds often builds up a stable layer at the surface and up through the first $\sim 200 \mathrm{~m}$ of the lowest edge of the atmosphere. This shallow nocturnal surface layer prevents air ventilation and hence accumulates vehicular emissions.

The four sampling routes ("transects") were about 3 to $4 \mathrm{~km}$ long (1 to $2 \mathrm{~km}$ upwind and 2 to $2.5 \mathrm{~km}$ downwind of the freeways). Each aligned as close to perpendicular as possible to straight sections of freeway. The freeways were roughly perpendicular to prevailing winds and away from interchanges with other freeways or major arterials. Each transect ran along quiet, residential two-lane streets surrounded (as much as possible) with one-story single family houses (Fig. S1b). None of the chosen transects had direct freeway access; this greatly reduces interference from local high-emitting vehicles and traffic in general. Sampling transects passed: under the 101 freeway in Downtown Los Angeles (DTLA), under the 91 freeway in Paramount, over the I-110 freeway in Carson, and over the I-210 in Claremont (Fig. S2). 


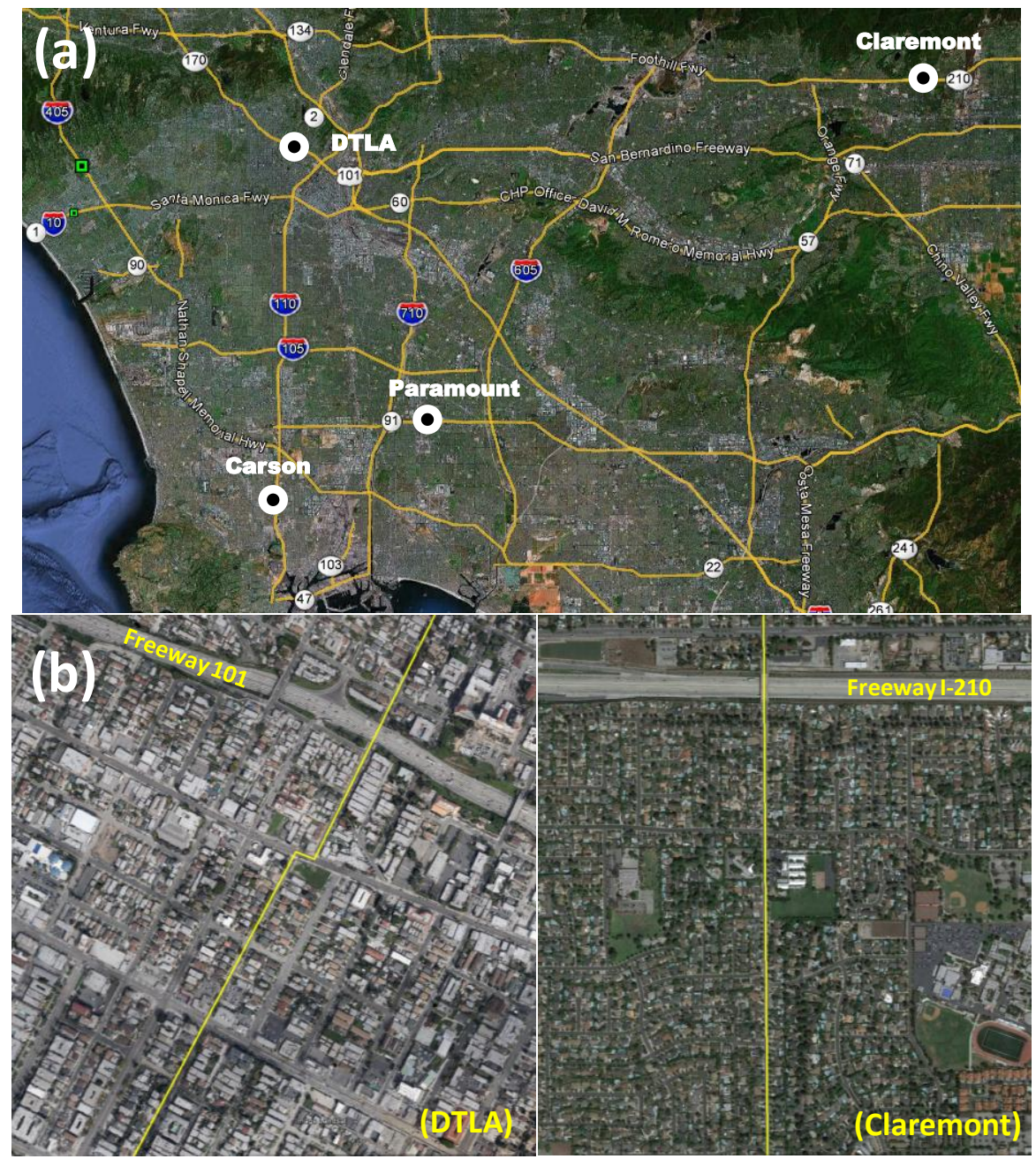

Fig. S1. (a) Map of transect locations where pre-sunrise measurements were conducted in the South California Air Basin (SoCAB). (b) Close up maps of transects on which the mobile platform was driven (yellow lines) and transect surroundings for in DTLA (bottom left) and Claremont (bottom right). Google Earth map.

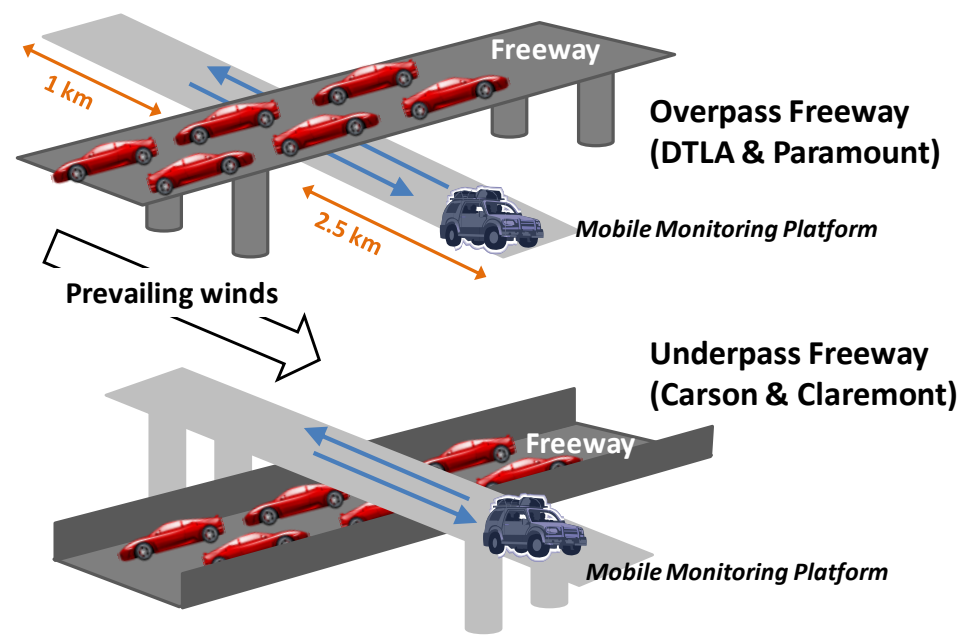

Fig. S2. Schematic illustration for freeway-transect geography for overpass (top) and underpass freeways (bottom). Sketch does not represent the scale of geographical features. 
The DTLA transect, near downtown Los Angeles, follows N. Coronado St., a small two lane street, running north-south. The entire upwind area and first $1500 \mathrm{~m}$ of the downwind area is residential. The farthest 1500 to $2200 \mathrm{~m}$ on the downwind side traverses a commercial district with tall buildings. The Paramount transect is located $11 \mathrm{~km}$ from the coast in a part of the coastal plain and is surrounded entirely by residential areas. The Carson transect is also on the coastal plain, $\sim 6 \mathrm{~km}$ northwest of the Ports of Los Angeles and Long Beach. The transect is mostly surrounded by residential areas, however the upwind end of the Carson (> $850 \mathrm{~m}$ from the freeway) and downwind ends of Paramount (> $1400 \mathrm{~m}$ from the freeway) are adjacent to industrial/commercial areas. We did not find evidence of pollutant emissions from these industrial areas in our measurements as might be expected particularly in the pre-sunrise hours. Finally, the Claremont transect is located in an inland valley, $70 \mathrm{~km}$ from the coast at the foot of steeply rising San Gabriel Mountains. The transect is entirely surrounded by quiet residential areas. The DTLA transect is crossed by several arterial streets downwind of the freeway: Temple

St., Beverly Blvd., $3^{\text {rd }}$ St., $6^{\text {th }}$ St., and Wilshire Blvd. The Carson, Paramount and Claremont transects each are crossed by just one or two major streets: Figueroa St. and Main St. for the Carson transect, Artesia Blvd. for the Paramount transect, and Foothill Blvd. for the Claremont transect. However, only small numbers of vehicles were observed on the cross streets during the pre-sunrise measurement periods. Nonetheless, to avoid possible interference from local vehicular emissions on these cross streets, data obtained in the vicinity (several tens meters on the downwind side) of these streets were excluded from our analyses. Some parts of the above descriptions were taken from Choi et al. (2012;2013).

\section{S2. Instrumentations, sampling, and post-data processing}

A Toyota RAV4 electric sub-SUV was used as mobile monitoring platform (MMP) to avoid self pollution from vehicle exhaust. The most significant UFP sources are the various combustion sources (Morawska et al., 2008). Although road-tire interaction, brake wear and re-suspension are known to be significant contributors to particle mass (particularly $\mathrm{PM}_{10}$ ), but their contributions to UFP are less well established (Kumar et al., 2013). Nonetheless, we note that UFP emissions from tire-road interaction and brake wear depend on the vehicle speed at which braking is initiated; and low vehicle speeds $(<30 \mathrm{~km} / \mathrm{h})$ emit very limited UFP (Kumar et al., 
2013): for road-tire interaction, Gustafsson et al. (2008) estimated 1,500 particles $\cdot \mathrm{cm}^{-3}$, which is one order of magnitude smaller than our background upwind concentrations; Mathissen et al. (2011) found no UFP formation for normal braking conditions and their upper limit estimate of UFP emissions from the road-tire interface is two orders of magnitude smaller than our estimate for mixed fleet combustion vehicles studied in the present study. Consistent with this, we do not observe either increases in UFP numbers nor changes in size distributions during driving, compared to when the MMP is stopped. Thus we believe the MMP did not affect observed UFP number concentrations in the present study.

The MMP was equipped with a suite of fast response instruments for various air pollutants: CPC 3007 and FMPS for ultrafine particle number concentrations; DustTraks for $\mathrm{PM}_{2.5}$ and $\mathrm{PM}_{10}$; PAS 2000 for particle-bound polycyclic aromatic hydrocarbons and instrumentation to monitor $\mathrm{CO}, \mathrm{NO}$ and $\mathrm{CO}_{2}$. The MMP was also equipped with a GPS (Garmin 76CS) for MMP position and a 2D-sonic anemometer for winds; and temperature and humidity sensors (Choi et al., 2012). Spatial distributions for pollutants other than UFP near the freeways were described in more detail in Choi et al. (2012). The same MMP has been used in a number of studies conducted in the SoCAB and the detailed instrumentation and calibration information is available elsewhere (Hu et al., 2009; Kozawa et al., 2012;Westerdahl et al., 2005;Choi et al., 2012). Briefly, air was pulled through a 6" diameter galvanized steel manifold installed through windows of the rear passenger space, facing the sidewalk $(1.5 \mathrm{~m}$ a.g.1.) by a fan located downstream of all sampling ports. Sampling ports for each instrument are located in the middle of manifold with short (0.5 to $2 \mathrm{~m}$ ) sampling tubing (1/4" Teflon for gases and 1/4" conductive tubing for particles and 1/2" conductive tubing for FMPS). Particle and gas instruments were calibrated by their respective manufacturers just before field measurements began. Calibration checks for gas-instruments were also conducted before each sampling campaign. Flow and zero checks were conducted on a daily basis. Data were recorded using a data-logger (Eurotherm Chessell Graphic DAQ Recorder) with 1 second time resolution, which corresponds to 5 to $8 \mathrm{~m}$ spatial resolution when the MMP was driving at 20 to $30 \mathrm{~km} / \mathrm{h}$, the normal driving condition during the measurements described here.

The MMP was driven along transects during pre-sunrise periods (4:30 to 6:30 LT). For most sampling days the last run was completed just before sunrise time, resulting in about 6 profiles 
(scans) per day in general. The sparse local traffic on the transect allowed the MMP to be driven at the same low speed (less than $30 \mathrm{~km} / \mathrm{h}$ ) along the whole transect, so that fine spatial resolution of concentration profiles could be obtained ( 5 to $8 \mathrm{~m}$ ). Once sampling was completed, the crosscorrelation method (Eq. S1, Choi et al., 2012) was applied on a daily basis to correct the different response time of each instrument in the MMP, which was caused by the characteristics of the instruments themselves and the length and flow rates through their inlets. Several smoke tests were also conducted as a reference. The cross-correlation method is based on the following expression:

$$
r=\frac{1}{T \sigma_{a} \sigma_{b}} \int_{t_{0}}^{t_{0}+T}(a(t)-\bar{a})(b(t+\tau)-\bar{b}) d t
$$

where $a$ and $b$ are simultaneously measured species, $t$ is time, $\tau$ is a time-lag applied to time series in $b, \sigma$ is the standard deviation for the two pollutants $a$ and $b$, and $T$ is the number of data points in the time-series. Data synchronization using cross-correlation is effective because traffic-related pollutants are emitted concomitantly from vehicles and reach peak concentrations near the sources, e.g., major roadways (Choi et al., 2012). After synchronizing instrument response times, local transient spikes in spatial concentration profiles from nearby high-emitting vehicles were removed by a running low $25 \%$ quantile method with varying window sizes (Choi et al., 2012): $53 \mathrm{~s}$ for distances farther than $1 \mathrm{~km}$ from the freeway; $31 \mathrm{~s}$ for distances between $300 \mathrm{~m}$ and $1 \mathrm{~km}$; and $3 \mathrm{~s}$ within $300 \mathrm{~m}$ from the freeways. This method successfully removed transient local spikes without altering remaining data. We additionally examined any remaining local effects, particularly near freeways, by reviewing video and audio records to verify proximity of high emitting vehicles before removing corresponding data.

A balloon tether sonde (SmartTether ${ }^{\mathrm{TM}}$, Anasphere Inc.) was used to probe the vertical temperature, humidity and wind gradients to determine atmospheric stability. Vertical profiles (up to $100 \mathrm{~m}$ a.g.1.) for temperature, humidity and winds were obtained on a daily basis (about 30 minutes before the MP measurements) near the transects (560 m away from the Downtown LA transect, $1.2 \mathrm{~km}$ from the Paramount transect, $3.7 \mathrm{~km}$ from the Carson transect, and $3.8 \mathrm{~km}$ from the Claremont transect). It was not possible to launch the balloon immediately adjacent to the transects due to air safety regulations (balloon launches are prohibited within $8 \mathrm{~km}$ of any airport) as well as the requirement for adequate open space to launch a balloon. 


\section{S3. General Meteorology and Traffic Conditions for Measurement Periods}

The usual prevailing wind direction was approximately perpendicular to the freeway for the DTLA, Paramount, and Carson transects with mean directions in the 73 to $82^{\circ}$ range relative to the freeways $\left(90^{\circ}\right.$ being normal to the freeway orientation). For the Claremont transect, winds were more askew to the freeway with a mean direction of $58^{\circ}$. Winds for this transect were the least variable however, due to the adjacent mountains to the north which produce a strong, thermally induced, mountain-valley wind system. Wind speeds during the sampling periods were generally calm ( 0.3 to $1.1 \mathrm{~m} \cdot \mathrm{s}^{-1}$ for all sampling days). Investigated areas were influenced by weak off-shore breezes during our sampling periods; the transition to the stronger daytime onshore wind regime generally occurs around 9 A.M. in the summer and later in the winter, well after the completion of our measurements. Temperature varied day-by-day, ranging from 3 to 15 ${ }^{\circ} \mathrm{C}$, but varied little (within $\pm 0.5^{\circ} \mathrm{C}$ ) during our relatively short early morning sampling periods. Static atmospheric stability can be represented with a vertical potential temperature gradient $(\mathrm{d} \Theta / \mathrm{dz}>0$ for stable, $\mathrm{d} \Theta / \mathrm{dz} \sim 0$ for neutral, and $\mathrm{d} \Theta / \mathrm{dz}<0$ for unstable). During the measurement periods, $d \Theta / d z$ was slightly positive for all transects. indicating slightly stable conditions. The vertical temperature gradient was highest near the Claremont transect $\left(1.23 \times 10^{-2}\right.$ $\mathrm{K} \cdot \mathrm{m}^{-1}$ ) although the differences between locations were not significant. Winds were generally calm with little vertical gradient during the measurements periods although the Claremont transect showed relatively stronger wind gradient compared to the other sites, making the lower edge of the atmosphere more neutral in terms of Richardson number (Fig. 8b).

The MMP measurements were conducted during the period of sharply increasing traffic flow on the freeways due to the onset of the morning commute. The 5 minute traffic and truck flows on the freeways were obtained from the Freeway Performance Measurement System (PeMS) sensors in the vicinity of the sampling transects: $100 \mathrm{~m}$ northeast of the DTLA transect (VDS ID: 717452); 550 m east of the Paramount transect (VDS ID: 765467); $850 \mathrm{~m}$ south of the Carson transect (VDS ID:763522); and $60 \mathrm{~m}$ east of the Claremont transect (VDS ID:767984). The mean traffic flows during the measurement periods were 800,1000,630, and 470 vehicles per 5 minutes on the 101 (DTLA), 91 (Paramount), I-110 (Carson), and I-210 freeways (Claremont), respectively. The fleet mixes on the transects were not characterized in detail; however they were not obviously different from one another. Truck flows accounted for a small fraction of the total 
traffic flows, falling in a similar range for all transects (2.4 to 6\%, Table 3 in Choi et al. (2012)). The differences in truck contribution should result in moderate differences in mixed-fleet emission rates for each transect, as well as between our measurements and those in the literature.

\section{S4. Variations in fitting parameters $\left(Q_{c}, \alpha\right.$, and $\left.\beta\right)$ in pollutant concentration profiles and expected relationships between them}

In Section 2.3.2, we discuss how the spatial concentration profiles change as fitting parameters $\left(Q_{c}, \alpha\right.$ and $\left.\beta\right)$ are varied. Here we discuss this issue in more detail, and consider if relationships should be expected between fitting parameters arising from the mathematical relation itself (independent of the physical processes that alter the spatial concentration profiles).

Given that the wind-corrected emission factor $\left(Q_{c}\right)$ is not a function of $x$ (horizontal distance from the freeway) in Eq. (2) (we assume that freeways are the only emission sources), then the dilution factor $f$ (Zhang and Wexler, 2004), the ratio of concentrations at distance $x$ to the peak, is only a function of $\sigma_{z}$ and the source height ( $\mathrm{z}-H$ and $\left.z+H\right)$ :

$$
f=\frac{\Delta C(x, z)}{\Delta C\left(x_{p}, z\right)}=\frac{\sigma_{z\left(x_{p}\right)}}{\sigma_{z(x)}} \frac{\exp \left(-\frac{(z-H)^{2}}{2 \sigma_{z(x)}^{2}}\right)+\exp \left(-\frac{(z+H)^{2}}{2 \sigma_{z(x)}^{2}}\right)}{\exp \left(-\frac{(z-H)^{2}}{2 \sigma_{z\left(x_{p}\right)}^{2}}\right)+\exp \left(-\frac{(z+H)^{2}}{2 \sigma_{z\left(x_{p}\right)}^{2}}\right)}
$$

where $\Delta C$ is background-subtracted concentration, and $x_{p}$ is distance at which concentration peaks. Thus $Q_{c}$ should be independent of relative concentration decay rates.

The partial derivative with respect to $x$ of the modified Gaussian expression is a complicated, multi-termed third order exponential function without any simple relationships, however, as expected from the form of Eq. 2, $Q_{c}$ appears only as a coefficient. Setting the partial derivative equal to zero to find the location of the peak concentration, $\triangle U F P_{\text {peak }}$, and simplifying this expression, removes $Q_{c}$ altogether (Eq. S3). Thus, $Q_{c}$ is mathematically unrelated to the location of the plume peak, which is most sensitive to $\alpha$, and emission rates should only determine the overall magnitude of freeway plumes:

$$
\frac{\partial C}{\partial x}=\frac{1}{\sigma_{z} \cdot x}\left[e^{\left(\frac{-H_{1}}{\sigma_{z}^{2}}\right)}\left(\frac{2 H_{1}}{\sigma_{z}^{2}}-\frac{2 H_{1} \beta}{\sigma_{z} \cdot \alpha}-\frac{1}{1+\beta x}\right)+e^{\left(\frac{-H_{2}}{\sigma_{z}^{2}}\right)}\left(\frac{2 H_{2}}{\sigma_{z}^{2}}-\frac{2 H_{2} \beta}{\sigma_{z} \cdot \alpha}-\frac{1}{1+\beta x}\right)\right]=0
$$


where:

$\sigma_{z}=\frac{\alpha \cdot x}{(1+\beta x)}, \quad H_{1}=\frac{(z+H)^{2}}{2}$, and $H_{2}=\frac{(z-H)^{2}}{2}$.

At a fixed specific distance $x_{1}$, concentration $C\left(x_{1}\right)$ depends on $\sigma_{z}\left(\alpha, \beta\right.$; Eq. 3) as well as $Q_{c}$. Figure S3(a) illustrates how $C\left(x_{1}\right)$ with constant $Q_{c}$ varies as a function of $\sigma_{z}(\alpha, \beta)$. However, the absolute and relative variations in $C\left(x_{1}\right)$ obviously depend on $x_{1}$ (e.g., how far is $x_{1}$ located from the peak as shown in Fig. S3b). With a fixed $Q_{c}$, the absolute concentration at the peak does not change as $\alpha$ and/or $\beta$ are varied (Figs. S3b and S3c). Figure S3c shows more clearly how concentration profiles change with respect to $Q_{c}, \alpha$ and $\beta$. From this figure we can see graphically that $Q_{c}$ should be determined only by the background subtracted peak concentration $\triangle[U F P]_{\text {peak }}$ and is mathematically independent of $\alpha$ and $\beta$. Consequently, the strong relationships between $\Delta[U F P]_{\text {peak }}$ (and hence $Q_{c}$ ) and $\alpha$ and $\beta$ found in our fitting results are not due to mathematical relationships in the fitting function, arising instead from physical processes as discussed in Section 3.4.1.

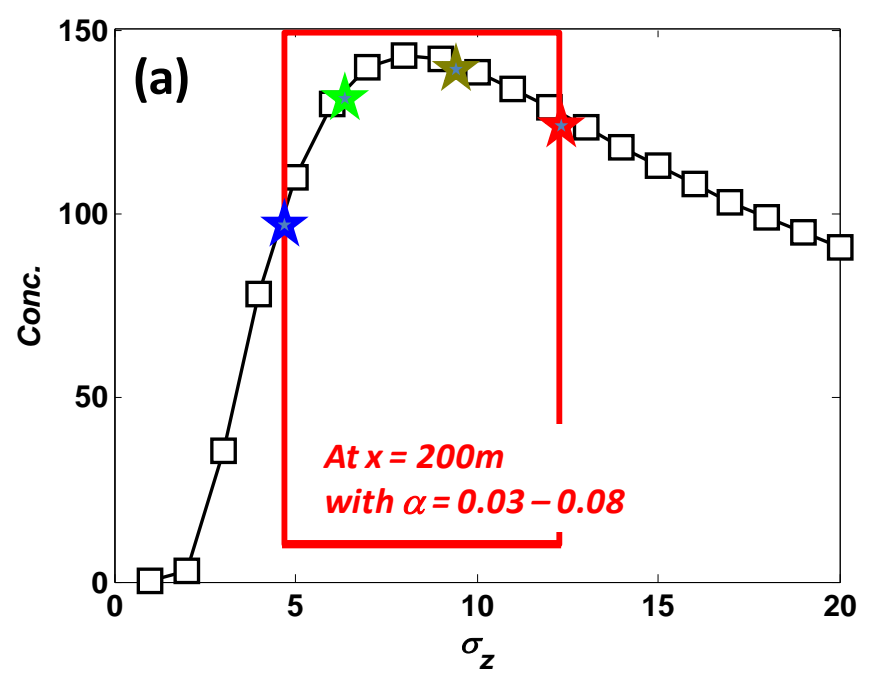

Figure S3. (a) Concentration variations in arbitrary units as a function of $\sigma_{z}$ at fixed distance $x_{1}=200 \mathrm{~m} . Q_{c}$ and $\beta$ are also fixed, and $\alpha$ varies from 0.03 to 0.08 [as shown in the red box], (b) concentration profiles with distance from the freeway $(x)$ under the same conditions as (a). Stars in (b) at distance $x_{1}=200 \mathrm{~m}$ correspond to the same symbols in (a), and (c) concentration profiles in arbitrary units with varying $Q_{c}, \alpha$, and $\beta$. 

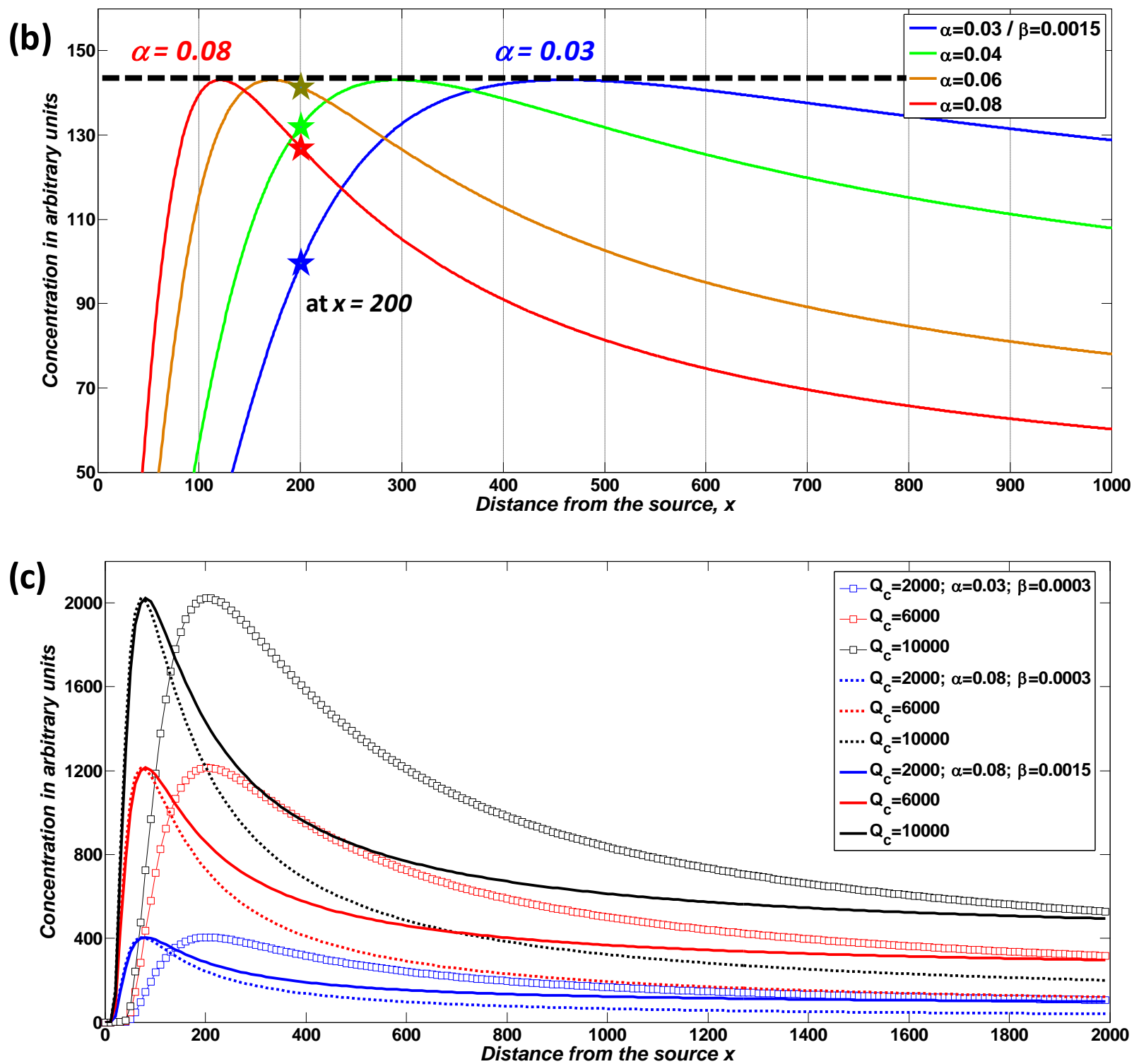

We do also not find any relationships between $\alpha$ and $\beta$ mathematically built into the fitting function. Because $\alpha$ and $\beta$ have different orders of magnitude, they each play a dominant role in determining $\sigma_{z}$ in different distance ranges (Eq. 3). For example, $\alpha$ plays the dominant role within $200 \mathrm{~m}$ from the freeway because $\alpha$ is one order of magnitude higher than $\beta$. The importance of $\beta$, however, increases with distance (Fig. S4). Thus, a combination of $\alpha$ and $\beta$ to best represent the peak location and downwind concentrations is determined in the fitting processes to the observed profiles, not by mathematics built in the fitting function. 


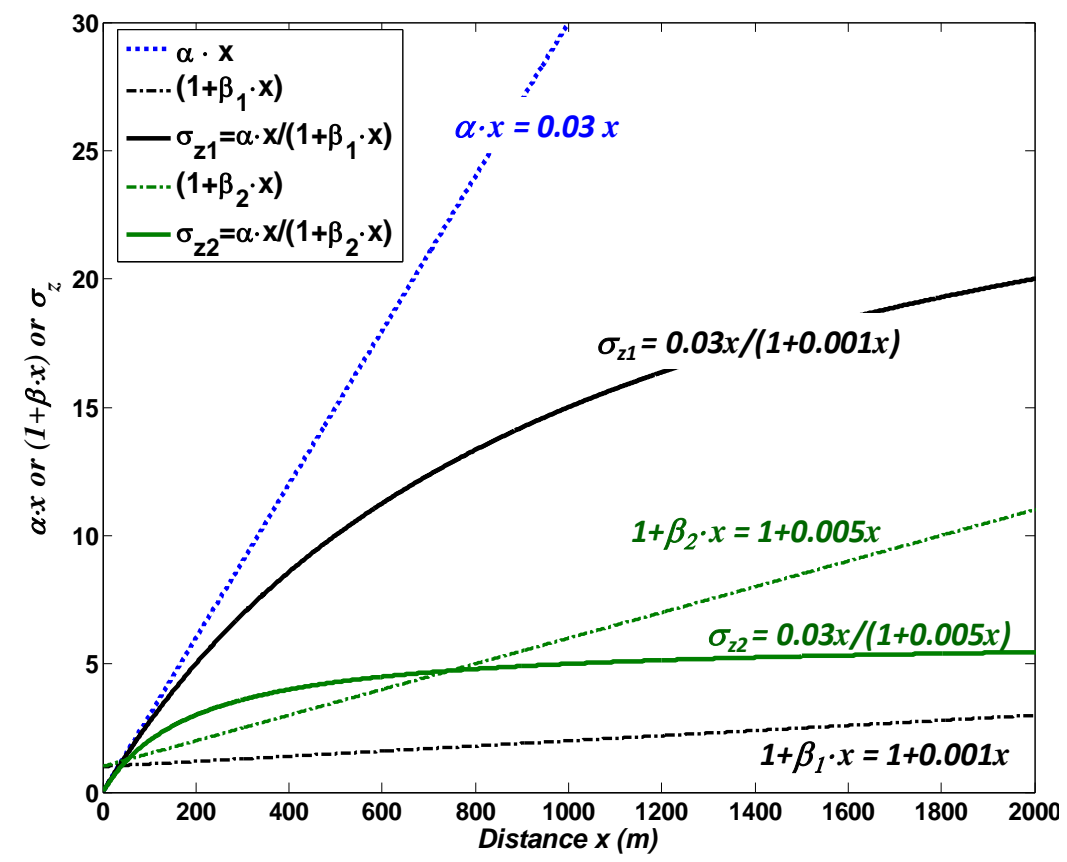

Figure S4. $\sigma_{z}$ and a numerator $(\alpha \cdot x ;$ blue dotted line) and denominator $(1+\beta \cdot x$; black and green dash-dotted lines) in $\sigma_{z}$ (black and green solid lines) as a function of distance $x$. $\alpha=0.03, \beta_{1}=0.001$ and $\beta_{2}=$ 0.005 .

\section{S5. Evaluation of multivariate regression (MVR) model results}

As indicated in the manuscript, we do not have enough data to establish a firm multivariate regression results and to evaluate those results with a test dataset held separately from training dataset. Thus, here we attempt to estimate the errors associated with the MVR analyses. First, we obtained the MVR coefficients with $(n-1)$ data points, holding one data point to be compared with an estimate from the MVR results. This process was iterated $n$ times: 10 times for overpass freeway transects (for $\alpha$ estimates) and 13 times for underpass freeways (for $\beta$ estimates). Second, the $\beta$ values for overpass freeways and $\alpha$ values for underpass freeways were calculated from the $\alpha-\beta$ relationships presented in the manuscript (Eqs. 10 and 11). Thus, estimated $\alpha$ and $\beta$ values are not involved in the MVR training processes. In general, the training results with $(n-1)$ dataset were similar with the results from the whole dataset presented in the manuscript (Fig. S5a), and estimated $\alpha$ and $\beta$ values showed good agreement with fitted values, although there were a few outliers (Fig. S5b). 

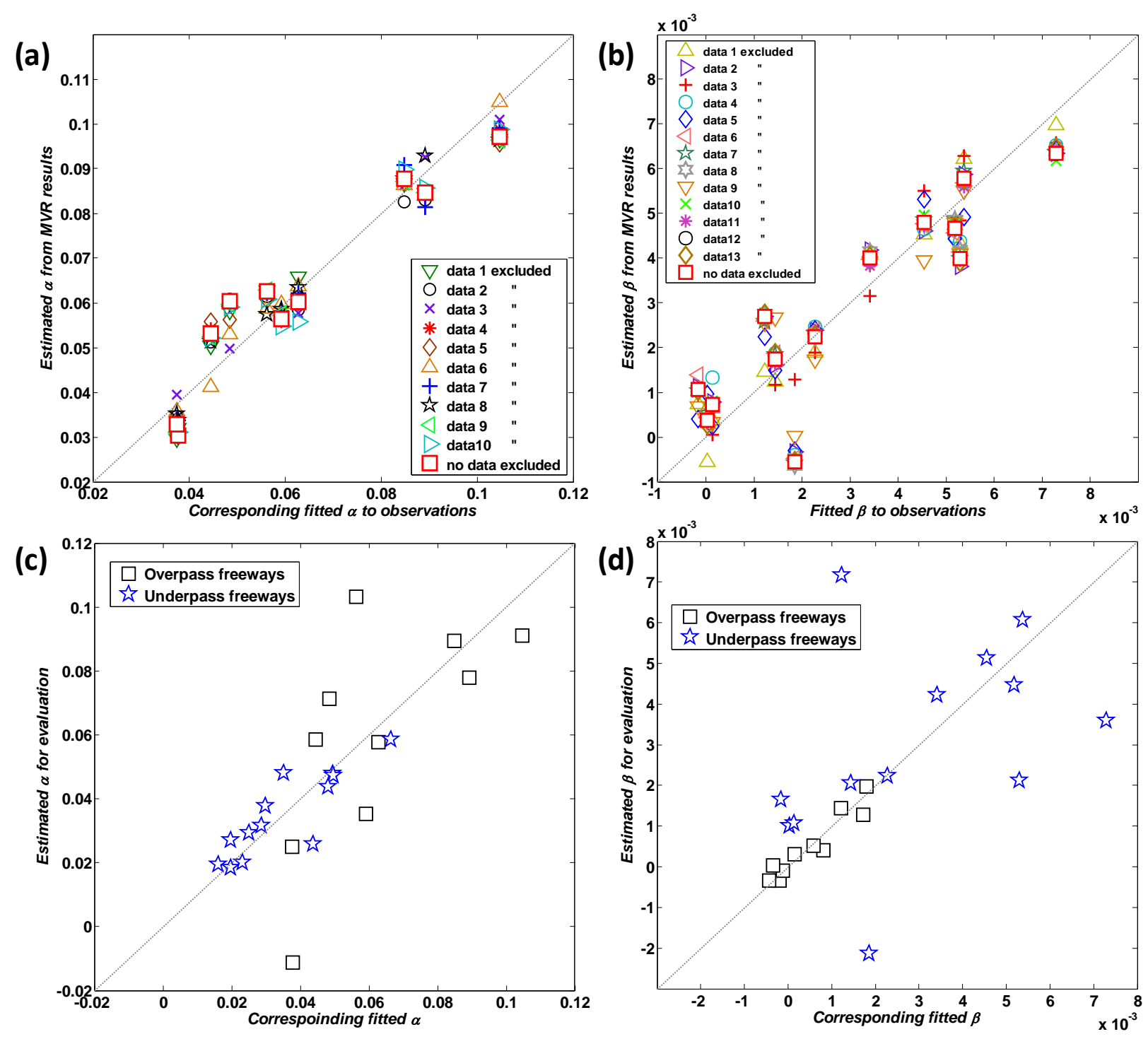

Figure S5. Comparisons between estimated $\alpha$ and $\beta$ values from MVR and the corresponding best-fit values from the original data. (a) and (b) show comparisons of training results for $n-1$ multivariate regressions (from which one data point has been excluded from each trial, indicated in the legend) for $\alpha$ (overpass freeways) and $\beta$ (underpass freeways) respectively. (c) and (d) show the ability of the MVR to predict the 'held back' data points; for $\alpha$ and $\beta$, respectively (see text). The $\alpha$ values for underpass freeways and $\beta$ values for overpass freeways were obtained from the relationships in Eqs. (10) and (11).

The overall errors from our best estimate are \pm 0.018 for $\alpha$ and \pm 0.0018 for $\beta$, based on linear regression analyses of the data shown in Figs. S5(c) and (d). These errors correspond to $26 \%$ and $60 \%$ for $\alpha$ and $\beta$, respectively when we define an error as the mean deviation from the fitted values over the mean value (Eq. S4): 


$$
\text { error }=\frac{\sum\left|a_{E S T}-a_{F i t}\right|}{N \cdot \overline{a_{F i t}}} \times 100
$$

where $a$ is an arbitrary symbol for data, the subscript EST denotes an estimate from MVR results, Fit represents the corresponding fitted value, and $N$ is number of data. As might be expected, a one or two of outliers have a large influence on the performance of the MVR, due to the limited size of the training dataset.

\section{References}

Choi, W., He, M., Barbesant, V., Kozawa, K. H., Mara, S., Winer, A. M., and Paulson, S. E.: Prevalence of wide area impacts downwind freeways under pre-sunrise stable atmospheric conditions, Atmospheric Environment, 62, 318-327, http://dx.doi.org/10.1016/j.atmosenv.2012.07.084, 2012.

Choi, W., Paulson, S. E., Casmassi, J., and Winer, A. M.: Evaluating meteorological comparability in air quality studies: classification and regression trees for primary pollutants in California's South Coast Air Basin, Atmospheric Environment, 64, 150-159, http://dx.doi.org/10.1016/j.atmosenv.2012.09.049, 2013.

Hu, S. S., Fruin, S., Kozawa, K., Mara, S., Paulson, S. E., and Winer, A. M.: A wide area of air pollutant impact downwind of a freeway during pre-sunrise hours, Atmospheric Environment, 43, 2541-2549, 10.1016/j.atmosenv.2009.02.033, 2009.

Kozawa, K. H., Winer, A. M., and Fruin, S. A.: Ultrafine particle size distributions near freeways: Effects of differing wind directions on exposure, Atmospheric Environment, 63, 250-260, 2012.

Kumar, P., Pirjola, L., Ketzel, M., and Harrison, R. M.: Nanoparticle emissions from 11 nonvehicle exhaust sources - A review, Atmospheric Environment, 67, 252-277, 10.1016/j.atmosenv.2012.11.011, 2013.

Morawska, L., Ristovski, Z., Jayaratne, E. R., Keogh, D. U., and Ling, X.: Ambient nano and ultrafine particles from motor vehicle emissions: Characteristics, ambient processing and implications on human exposure, Atmospheric Environment, 42, 8113-8138, 10.1016/j.atmosenv.2008.07.050, 2008.

Westerdahl, D., Fruin, S., Sax, T., Fine, P. M., and Sioutas, C.: Mobile platform measurements of ultrafine particles and associated pollutant concentrations on freeways and residential streets in Los Angeles, Atmospheric Environment, 39, 3597-3610, 10.1016/j.atmosenv.2005.02.034, 2005.

Zhang, K. M., and Wexler, A. S.: Evolution of particle number distribution near roadways - Part I: analysis of aerosol dynamics and its implications for engine emission measurement, Atmospheric Environment, 38, 6643-6653, 10.1016/j.atmosenv.2004.06.043, 2004. 